(с) Н.А. Зубкова', О.А. Гиоева', В.М. Петров', Е.В. Васильев', А.В. Тимофеев², А.В. Абрукова, А.Н. Тюльпаков

'ФГБУ Национальный медицинский исследовательский центр эндокринологии Минздрава России, Москва

${ }^{2}$ ФГАОУ ВО Первый МоскОВский государственный медицинский университет имени И.М. Сеченова Минздрава России (Сеченовский университет), Москва

БУ Президентский перинатальный центр Минздрава Чувашии, Чебоксары

Сахарный диабет типа MODY - генетически и клинически гетерогенная группа заболеваний, характеризующаяся аутосомнодоминантным типом наследования и обусловленная мутациями генов, приводящими к дисфункции $\beta$-клеток поджелудочной железы. К настоящему времени верифицировано 13 подтипов MODY, наиболее распространенными из которых являются подтипы 1-3. MODY2 и MODY3 ранее неоднократно описаны в нашей стране. Описания более редких подтипов МODY в отечественной литературе единичны. В данной работе мы приводим клиническое и молекулярно-генетическое описание двух впервые выявленных в России случаев редкого подтипа MODY, обусловленного дефектом гена PAX4 (MODY9). Ген PAX4 кодирует фактор транскрипции PAX4, который участвует в дифференцировке $\beta$-клеток поджелудочной железы. Мутации в гене РАХ4 приводят не только к нарушению развития $\beta$-клеток поджелудочной железы, но и снижению процессов их регенерации в последующей взрослой жизни. Молекулярно-генетическое подтверждение диагноза проведено с использованием технологии секвенирования нового поколения, позволяющей проводить одновременный анализ нескольких генов-кандидатов. Данная технология активно внедрена в последнее время в отечественную практику для генетической верификации моногенных заболеваний и, в частности, MODY. Найденные мутации ранее не описаны.

кЛЮчЕВЫЕ СЛОВА: сахарный диабет; гестационный сахарный диабет; MODY; PAX4; MODY9; NGS

\title{
MONOGENIC DIABETES ASSOCIATED WITH PAX4 GENE MUTATIONS (MODY9): FIRST DESCRIPTION IN RUSSIA
}

(c) Natalya A. Zubkova', Olesya A. Gioeva', Vasily M. Petrov', Evgeny V. Vasilyev', Alexei V. Timofeev², Anna V. Abrukova ${ }^{3}$, Anatoly N. Tiulpakov

'Endocrinology Research Centre, Moscow, Russia

${ }^{2}$ I.M.Sechenov First Moscow State Medical University, Moscow, Russia

${ }^{3}$ Presidential Perinatal Centre, Cheboksary, Russia

Maturity-onset diabetes of the young (MODY) is a heterogeneous group of disorders characterised by autosomal dominant type of inheritance and caused by genetic defects leading to dysfunction of pancreatic beta-cells. To date, at least 13 subtypes of MODY have been described in the literature, the most frequent of which are MODY types 1-3. MODY2 and MODY3 are the most prevalent subtypes, and were previously described in our country, Russia. Several cases of rare MODY subtypes were subsequently described in the Russian literature. The current report is the first in the Russian literature to present clinical and molecular genetic characteristics of two cases of another rare MODY subtype-MODY9. This type of MODY is associated with mutations in the PAX4 gene, which encodes transcription factor PAX4, one of the factors essential for pancreatic beta-cell differentiation. Molecular genetic analysis was performed using next-generation sequencing, a new method recently applied to verify monogenic diseases and, in particular, MODY. This study reports a novel mutation in the PAX4 gene in MODY patients.

KEYWORDS: maturity-onset diabetes of the young; gestational diabetes mellitus; MODY; PAX4; MODY9; NGS

\section{ВВЕДЕНИЕ}

Изучение молекулярных основ эндокринных заболеваний является приоритетным направлением в эндокринологии. Подтверждение моногенного характера заболевания позволяет четко регламентировать характер наблюдения пациента, назначить патогенетическую терапию и планировать рождение здорового потомства. С 1974 г., когда впервые были описаны семьи с доминантно-наследуемым сахарным диабетом (СД), открыто около 30 генов, ответственных за развитие моногенных форм диабета. Распространенность наследственных форм диабета активно изучается среди различных групп пациентов, прежде всего потому, что именно у этих больных возможна компенсация углеводного обмена без назначения инсулинотерапии. В конечном итоге это позволяет снизить расходы на обеспечение и лечение пациентов.

Моногенные формы СД обусловлены, прежде всего, дефектами генов, регулирующих функцию $\beta$-клеток поджелудочной железы (ПЖ), к которым относятся подтипы диабета MODY («maturity-onset diabetes of the young», «диабет зрелого типа у молодых»). Истинная распространенность 13 подтипов MODY, открытых к настоящему времени, неизвестна. Большинство публикаций посвящено описанию мутаций в генах HNF1A, GCK и HNF4A, которые превалируют среди MODY. Мутации в других генах-кандидатах 
MODY редки и описаны лишь в отдельных семьях. Иными словами, сведения о фенотипе более редких форм крайне ограничены. Все вышесказанное делает чрезвычайно актуальным изучение молекулярных основ диабета. Однако выполнение этой задачи до недавнего времени было затруднительно в связи с большой трудоемкостью и дороговизной молекулярно-генетических исследований. Внедрение в практику высокопроизводительного параллельного секвенирования (next generation sequencing, NGS) позволяет проводить диагностический поиск одновременно среди десятков генов-кандидатов, что значительно упрощает постановку диагноза. Данная методика внедрена в отечественную практику и ранее нами описаны случаи MODY с дигенным и олигогенным наследованием [1] и случай MODY6 [2], выявленные с использованием NGS.

В настоящем сообщении речь пойдет об одном из редких вариантов MODY, обусловленном гетерозиготными мутациями в гене РАХ4, которые приводят к развитию диабета типа MODY9. Молекулярно-генетическое исследование проведено с использованием технологии NGS.

Ген PAX4 (paired box gene 4, paired domain gene 4) является членом семейства гомеобоксных РАХ генов [3], картирован на длинном плече хромосомы 7 в положении 32.1 (7q32.1) [4, 5], имеет 10 экзонов. Ген кодирует транскрипционный фактор PAX4, который, как и другие факторы транскрипции, участвует в дифференцировке $\beta$-клеток ПЖ. Кроме того, РАХ4 принимает участие и в дифференцировке d-клеток ПЖ. Роль РАХ4 в развитии $\beta$-клеток ПЖ доказана с использованием нокаута данного гена. У мышей с полным отсутствием гена РАХ4 $\beta$ - и d-клетки ПЖ не дифференцируются, но присутствуют а-клетки. Таким образом, главной функцией гена РАХ4 является контроль развития $\beta$ - и d-клеток ПЖ после активации гена NGN3. Также PAX4 регулирует экспрессию генов PDX1 и Nkx 6.1, участвующих в развитии ß-клеток ПЖ [6].

Мутации в гене РАХ4 приводят не только к нарушению развития $\beta$-клеток, но и снижению процессов их регенерации в последующей взрослой жизни, в связи с чем MODY9 характеризуется прогрессирующим снижением уровня инсулина и возможным развитием сосудистых осложнений.

Первые сообщения в литературе, в которых отображена связь мутаций в гене РАХ4 с СД, начали публиковаться в начале 2000-х годов, когда сообщалось об ассоциации мутаций в гене PAX4 с СД 2 типа [7, 8]. Лишь в 2007 г. авторы исследования, проведенного в Тайланде [9], впервые высказали предположение о взаимосвязи мутаций в гене PAX4 с MODY. Ими был исследован ген РAX4 у 46 пробандов с фенотипом MODY и найдены 2 новые патогенные мутации
(p.R164W и IVS7-1G>A). В дальнейшем описаны отдельные семьи с MODY9, также подтвержденного методом прямого секвенирования. Так, фенотип MODY9 описан в японской семье у 15-летнего пробанда и его отца [10]. Пробанд, не страдающий ожирением, в дебюте имел полиурию, полидипсию, гипергликемию до 35 ммоль/л, гликированный гемоглобин ( $\left.\mathrm{HbA}_{1 c}\right)$ 14,5\%, кетонурию, сохранную, но недостаточную секрецию инсулина, что потребовало назначения инсулинотерапии, тогда как диабет отца диагностирован в возрасте 30 лет на фоне ожирения и успешно компенсирован на диете. Однако отрицательный титр антител к GADA и ICA у пробанда наряду с отягощенной по диабету наследственностью послужили поводом для проведения молекулярно-генетического исследования, по результатам которого была найдена новая мутация с.374_412del39 в экзоне 3 гена PAX4. Аналогичная мутация найдена и у отца.

К настоящему времени в литературе, включая исследования, проведенные с использованием NGS [11-15], описано 8 мутаций в гене PAX4: 6 миссенс-мутаций, 1 мутация сайта сплайсинга и 1 протяженная делеция.

В рамках исследования, посвященного изучению структуры MODY в России, молекулярно-генетический анализ 13 генов-кандидатов MODY с использованием технологии NGS проведен 312 пациентам в возрасте от 3 мес до 25 лет. Критерии включения в исследование: нарушения углеводного обмена (НУО) различной степени выраженности, отрицательный титр аутоантител к ICA, GADA, IA-2, сохранная секреция эндогенного инсулина. Методика исследования подробно описана в статье, посвященной первому описанию MODY6 в России [2].

В гене РАХ4 мутации выявлены у 2 неродственных пробандов. Выявленные мутации ранее не описаны (табл. 1).

\section{ОПИСАНИЯ КЛИНИЧЕСКИХ СЛУЧАЕВ}

Клинический случай 1

У пациента 6,9 лет впервые гипергликемия натощак 6,2 ммоль/л выявлена в возрасте 6,2 лет в связи с жалобами на полидипсию и повышенную утомляемость. Повторно гликемия натощак 6,5 ммоль/л, через 2 ч после еды 6,3 ммоль/л; НbА 1 8,5\%; аглюкозурия. Рекомендовано соблюдение низкоуглеводной диеты. Через 3 мес обследован в стационаре: гликемия капиллярной крови натощак в пределах 4,4-4,9 моль/л, в течение дня максимально до 6,5 ммоль/л (отмечалось лишь однократное повышение гликемии венозной крови натощак до 7,9 ммоль/л); $\mathrm{HbA}_{1 с}$ 7,3\%; стойкая аглюкозурия. При осмотре обращал на себя внимание избыток массы тела (SDS ИMT +1,2). В ходе пе-

Таблица 1. Спектр выявленных мутаций в гене PAX4

\begin{tabular}{ccccc}
\hline Пациент & Нуклеотидная замена & Аминокислотная замена & Тип мутации & Экзон \\
\hline 1 & с.55C>T & p.R19W & миссенс \\
2 & c.596C>T & p.T199l & миссенс & 5 \\
\hline
\end{tabular}

Таблица 2. Показатели ПГТТ пациента с мутацией p.R19W

\begin{tabular}{lcccc}
\hline \multicolumn{1}{c}{ Показатель } & 0 мин & 30 мин & 60 мин & 120 мин \\
\hline Глюкоза, ммоль/л & 5,7 & 9,0 & 6,1 & 7,0 \\
С-пептид, нг/мл & 1,09 & 6,91 & 5,78 & 11,89 \\
Инсулин, мкМЕ/мл & 6,76 & 55,16 & 17,16 & 24,46 \\
\hline
\end{tabular}


Таблица 3. Показатели ПГТТ пациента с мутацией р.Т199I

\begin{tabular}{cccccc}
\hline Показатель & 0 мин & 30 мин & 60 мин & 90 мин & 120 мин \\
\hline Глюкоза (венозная плазма), ммоль/л & 4,93 & 9,48 & 8,92 & 8,26 & 6,55 \\
\hline
\end{tabular}

рорального глюкозотолерантного теста (ПГТТ) выявлена умеренная пограничная гипергликемия натощак (ПГН), адекватный тип инсулиновой секреции без инсулинорезистентности (индекс Matsuda 6,78 (норма >3), HOMA 1,71 (норма <3,2)) (табл. 2).

В ходе иммунологического исследования выявлен отрицательный титр маркеров СД 1 типа (антитела к GADA, ICA, |A-2).

Учитывая нетипичную клиническую картину диабета, отрицательный титр антител, отягощенную по НУО наследственность (мать - ПГН, гестационный диабет), пациент направлен на проведение молекулярно-генетического исследования, по результатам которого выявлена ранее неописанная гетерозиготная миссенс-мутация с.55C>T p.R19W в экзоне 1 гена PAX4. Аналогичная мутация была выявлена у матери пробанда.

\section{Клинический случай 2}

У пациента 16 лет с нормальной массой тела (SDS UMT -0,54) и отсутствием клинических признаков СД постпрандиальная гипергликемия 12,8 ммоль/л впервые выявлена в ходе диспансеризации. При обследовании в стационаре на фоне соблюдения низкоуглеводной диеты гликемия натощак за весь период госпитализации не превышала 5,05 ммоль/л, в течение дня - 7,6 ммоль/л, лишь однократно зафиксирована гликемия 9,2 ммоль/л через 2 ч после еды; НbА, 4,1\% (норма 4-6\%), С-пептид - 2,1 нг/мл (норма 0,81-3,83); аглюкозурия; титр аутоантител к ICA, IA-2, GADA отрицательный. В ходе ПГТТ НУО не выявлены (табл. 3).

Достоверные сведения о характере НУО у родственников уточнить не удалось по причине гибели родителей, однако известно, что у матери в возрасте 39 лет при случайном измерении гликемии диагностирован СД на фоне избыточной массы тела, потребовавший назначения инсулинотерапии. Бабушка по линии матери наблюдалась с СД, диагностированным в пожилом возрасте также на фоне избыточной массы тела, получала инсулинотерапию, и при длительности заболевания 10 лет осложнения выявлены не были. Дедушка по линии матери также страдал СД, получал инсулинотерапию.

Учитывая результаты обследования и данные семейного анамнеза, с целью исключения моногенной формы СД пациенту проведено молекулярно-генетическое исследование, в результате которого установлен диабет MODY9 выявлена ранее не описанная гетерозиготная мутация c.596C>T р.T199I в экзоне 5 гена PAX4.

Оба пациента взяты под динамическое наблюдение.

\section{ЗАКЛЮЧЕНИЕ}

Описанный нами впервые в отечественной литературе подтип СД MODY9 достаточно плохо изучен в связи с редкой частотой встречаемости. Имеющиеся в литературе единичные клинические описания не дают возможность установить четкую корреляцию генотип-фенотип среди пациентов с MODY9, так как клиническая картина, которую демонстрируют пациенты с MODY9, достаточно вариабельна не только внутри подтипа, но и среди родственников с аналогичной мутацией.

По клиническим описаниям, имеющимся в литературе, MODY9 часто манифестирует с симптомов, характерных для СД 1 типа, отражая декомпенсацию диабета и требуя назначения инсулинотерапии. Однако ряд пациентов c MODY9 даже после острого начала в последующем могут быть успешно компенсированы на диете. Так, в частности, оба наших пациента на момент проведения молекулярно-генетического исследования не нуждались в назначении инсулинотерапии, а соблюдение диеты пациентом с манифестным диабетом привело к значительному улучшению гликемического профиля. Несмотря на это, имеющиеся данные о прогрессирующем снижении инсулиновой секреции при мутациях в гене РАХ4 требуют тщательного динамического наблюдения за пациентами с целью раннего предотвращения сосудистых осложнений.

Включение гена PAX4 в панели генов СД при использовании технологии NGS позволит в будущем более активно выявлять случаи MODY9 среди пациентов с неуточненным СД.

\section{ДОПОЛНИТЕЛЬНАЯ ИНФОРМАЦИЯ}

Согласие пациентов. Законные представители пациентов дали авторам письменное согласие на публикацию медицинских данных в журнале «Сахарный диабет»

Финансирование исследования. Работа выполнена при содействии Российского научного фонда (грант РНФ №16-15-10408)

Конфликт интересов. Авторы декларируют отсутствие явных и потенциальных конфликтов интересов, связанных с публикацией настоящей статьи

Участие авторов. Н.А. Зубкова - концепция и дизайн исследования; Н.А. Зубкова, О.А. Гиоева, Е.В. Васильев, В.М. Петров, А.В. Тимофеев, А.В. Абрукова, А.Н. Тюльпаков сбор и обработка материала; Н.А. Зубкова - написание текста; А.Н. Тюльпаков - редактирование, утверждение финальной версии рукописи.

\section{СПИСОК ЛИТЕРАТУРЫ | REFERENCES}

1. Гиоева О.А., Зубкова Н.А., Тихонович Ю.В., и др. Клиническая и молекулярно-генетическая характеристика случаев MODY с дигенным и олигогенным наследованием, выявленных по результатам высокопроизводительного параллельного секвенирования // Проблемы Эндокринологии. - 2016. - Т. 62. - №6. C. 20-27. [Gioeva OA, Zubkova NA, Tikhonovich YV, et al. Clinical and molecular genetic characteristics of MODY cases with digenic and oligogenic inheritance as defined by targeted next-generation sequencing. Problems of Endocrinology. 2017;62(6):20-27. (In Russ.)] doi: 10.14341/probl201662620-27

2. Гиоева О.А., Колодкина А.А., Васильев Е.В., и др. Наследственный вариант сахарного диабета, обусловленного дефектом гена 
NEUROD1 (MODY6): первое описание в России // Проблемы Эндокринологии. - 2016. - Т. 62. - №3. - С. 16-20. [Gioeva OA, Kolodkina AA, Vasilyev EV, et al. Hereditary variant of diabetes mellitus caused by a defect of the NEUROD1 gene (MODY6): the first description in Russia. Problems of Endocrinology. 2016;62(3):16-20. (In Russ.)] doi: 10.14341/probl201662316-20

3. Dahl E, Koseki H, Balling R. Pax genes and organogenesis. Bioessays. 1997;19(9):755-765. doi: 10.1002/bies.950190905

4. Matsushita T, Yamaoka T, Otsuka S, et al. Molecular cloning of mouse paired-box-containing gene (Pax)-4 from an islet beta cell line and deduced sequence of human Pax-4. Biochem Biophys Res Commun. 1998;242(1):176-180. doi: 10.1006/bbrc.1997.7935

5. Inoue H, Nomiyama J, Nakai K, et al. Isolation of full-length cDNA of mouse PAX4 gene and identification of its human homologue. Biochem Biophys Res Commun. 1998;243(2):628-633. doi: 10.1006/bbrc.1998.8144

6. Wang J, Elghazi L, Parker SE, et al. The concerted activities of Pax4 and $\mathrm{Nk} \times 2.2$ are essential to initiate pancreatic beta-cell differentiation. Dev Biol. 2004;266(1):178-189. doi: 10.1016/j.ydbio.2003.10.018

7. Shimajiri Y, Sanke T, Furuta H, et al. A missense mutation of Pax4 gene (R121W) is associated with type 2 diabetes in Japanese. Diabetes. 2001;50(12):2864-2869. doi: 10.2337/diabetes.50.12.2864

8. Mauvais-Jarvis F, Smith SB, Le May C, et al. PAX4 gene variations predispose to ketosis-prone diabetes. Hum Mol Genet. 2004;13(24):3151-3159. doi: 10.1093/hmg/ddh341
9. Plengvidhya $\mathrm{N}$, Kooptiwut $\mathrm{S}$, Songtawee $\mathrm{N}$, et al. PAX4 mutations in Thais with maturity onset diabetes of the young. J Clin Endocrinol Metab. 2007;92(7):2821-2826. doi: 10.1210/jc.2006-1927

10. Jo W, Endo M, Ishizu K, et al. A novel PAX4 mutation in a Japanese patient with maturity-onset diabetes of the young. Tohoku J Exp Med. 2011;223(2):113-118. doi: 10.1620/tjem.223.113

11. Chapla A, Mruthyunjaya MD, Asha HS, et al. Maturity onset diabetes of the young in India - a distinctive mutation pattern identified through targeted next-generation sequencing. Clin. Endocrinol (Oxf). 2015;82(4):533-542.doi: 10.1111/cen.12541

12. Szopa M, Ludwig-Gałęzowska A, Radkowski P, et al. Genetic testing for monogenic diabetes using targeted next-generation sequencing in patients with maturity-onset diabetes of the young. Pol Arch Med Wewn. 2015;125(11):845-851. doi: 10.20452/pamw.3164

13. Ellard S, Lango Allen $\mathrm{H}$, De Franco $\mathrm{E}$, et al. Improved genetic testing for monogenic diabetes using targeted next-generation sequencing. Diabetologia. 2013;56(9):1958-1963. doi: 10.1007/s00125-013-2962-5

14. Alkorta-Aranburu G, Carmody D, Cheng YW, et al. Phenotypic heterogeneity in monogenic diabetes: the clinical and diagnostic utility of a gene panel-based next-generation sequencing approach. Mol Genet Metab. 2014;113(4):315-320. doi: 10.1016/j.ymgme.2014.09.007

15. Anık A, Catlı G, Abacı A, et al. Molecular diagnosis of maturity-onset diabetes of the young (MODY) in Turkish children by using targeted next-generation sequencing. J Pediatr Endocrinol Metab. 2015;28(11-12):1265-1271. doi: 10.1515/jpem-2014-0430

\section{ИНФОРМАЦИЯ ОБ АВТОРАХ [AUTHORS INFO]}

Зубкова Наталья Анатольевна, К.M.H. [Natalya A. Zubkova, MD, PhD]; адрес: Россия, 117036, Москва, ул. Дм.Ульянова, д. 11 [address: 11 Dm. Ulyanova street, Moscow, 117036 Russian Federation]; ORCID: http://orcid.org/0000-0002-1346-7545; eLibrary SPIN: 5064-9992; e-mail: zunata2006@yandex.ru.

Гиоева Олеся Анатольевна, аспирант [Olesya A. Gioeva, MD, PhD student]; ORCID: http://orcid.org/0000-0001-9189-4596; eLibrary SPIN: 4994-4126; e-mail: olesyasogma@mail.ru. Петров Василий Михайлович, к.х.H. [Vasily M. Petrov, PhD in Chemistry]; ORCID: http://orcid.org/0000-0002-0520-9132; eLibrary SPIN: 4358-2147; e-mail: petrov.vasiliy@gmail.com. Васильев Евгений Витальевич, к.б.н. [Evgeny V. Vasiliev, PhD in Biology]; ORCID: http://orcid.org/0000-0003-1 107362X; eLibrary SPIN: 5767-1569; e-mail: vas-evg@yandex.ru. Тимофеев Алексей Валентинович, к.б.н. [Alexei V. Timofeev, PhD in Biology]; ORCID: http://orcid. org/0000-0002-6861-9630; eLibrary SPIN: 1117-6599; e-mail: alvaltim@gmail.com. Абрукова Анна Викторовна, к.м.н. [Anna V. Abrukova, MD, PhD]; ORCID: http://orcid.org/0000-0002-2225-2773; eLibrary SPIN: 1966-4764; e-mail: metra2009@yandex.ru. Тюльпаков Анатолий Николаевич, д.м.н. [Anatoly N. Tyulpakov, MD, PhD]; ORCID: http://orcid.org/0000-0001-8500-4841; eLibrary SPIN: 8396-1798; e-mail: ant@endocrincentr.ru.

\section{ЦИТИРОВАТЬ:}

Зубкова Н.А., Гиоева О.А., Петров В.М., Васильев Е.В., Тимофеев А.В., Абрукова А.В., Тюльпаков А.Н. Наследственный вариант сахарного диабета, обусловленного дефектом гена PAX4(MODY9), - первое описание в России // Сахарный диабет. — 2017. — T. 20. — №5. — C. 384-387. doi: 10.14341/DM9322

\section{TO CITE THIS ARTICLE:}

Zubkova NA, Gioeva OA, Petrov VM, Vasilyev EV, Timofeev AV, Abrukova AV, Tiulpakov AN. Monogenic diabetes associated with PAX4 gene mutations (MODY9): first description in Russia. Diabetes mellitus. 2017;20(5):384-387. doi: 10.14341/DM9322 